\title{
THE LIFETIME FORECASTING OF MACHINE ELEMENTS BY FATIGUE STRENGTH CRITERION
}

\author{
Yuriy KHOMIAK ${ }^{1}$, Oleksandr KIBAKOV ${ }^{2}$, Stanislav MEDVEDEV ${ }^{2}$, \\ Ilya NIKOLENKO ${ }^{3}$, Victoria ZHEGLOVA ${ }^{1}$ \\ ${ }^{1}$ Odessa Polytechnic State University, Ukraine \\ ${ }^{2}$ Odessa National Maritime University, Ukraine, \\ ${ }^{3}$ Academy of Construction and Architecture, "V.I. Vernadsky Crimean Federal University" \\ E-mail:jomiak38@gmail.com, kibakov60@gmail.com, energia-09@mail.ru, vheglova@gmail.com
}

\begin{abstract}
The method for assessing the lifetime of structural elements by the criterion of fatigue strength is improved. It includes tests of laboratory material samples, the positions of the statistical theory of similarity of fatigue failure, calculation of the stress state by the finite elements method and takes into account the nature of cyclic loading with variable amplitude. A way for calculating the equivalence coefficient by the cycles $\mu_{m}$ is proposed, which takes into account the characteristics of fatigue strength and the cyclogram of the loading of the machine elements. Mathematical descriptions of integral distribution functions for typical loading modes (heavy, medium equiprobable, medium normal, light and especially light) are given and on their basis the corresponding loading cyclograms are obtained. The technique is implemented on the example of one of the main parts of an axial piston hydraulic machine (APHM) - a cylinder block (CB) made of tin bronze CuSn12. The possibilities of increasing the working pressure in the APHM by constructive and technological methods are analyzed. It is shown that in the manufacture of CB from high-strength antifriction brass $\mathrm{Zn} 28 \mathrm{Mn} 3 \mathrm{Co} 2$, it is possible to increase the operating pressure in the APHM to $40 \mathrm{MPa}$, which for heavy duty operation will provide a CB lifetime more than nine thousand hours.
\end{abstract}

Keywords: fatigue curve parameters, endurance limit, fatigue fracture similarity equation, typical loading conditions

\section{INTRODUCTION}

In this work, the lifetime of a part is understood as its operating time from the beginning of operation until the onset of the limiting state. The operating time is determined by the number of load change cycles $N_{\Sigma}$ or in units of time $t_{\Sigma}$ (hours). The destruction of a part under the influence of cyclic stresses believed as the limit state. The lifetime is the main indicator of the reliability of the part. It installed according to the results of operation during field tests or at diagnostic stands $[1,2]$. Such methods require a large investment of time and funds, and at the same time, they do not allow to set the resource of all critical parts. Therefore, there is a need primarily identify the most problematic units or parts of the machine and assess their service life. The main methods for assessing the lifetime of such technical objects are:

- life time tests (at universal or specialized stands or during trial operation) $[1,2]$;

- monitoring - forecasting based on the analysis of statistical data on the functioning of similar objects, taking into account the conditions of their work [3, 4];

- mathematical modeling of an object, taking into account regression phenomena during its operation;

- expert analysis;
- extrapolation of information about the state of the object, obtained during its diagnosis.

There are methods of destructive and nondestructive testing (diagnostics).

Destructive testing methods (DT- methods) are based on bringing the investigated object or its model to the limit state (destruction, limit wear, etc.). DT-methods used, in particular, to establish the mechanical properties of a material.

Non-destructive testing methods (NDTmethods) allow you to check the quality of an object without compromising its serviceability. These are ultrasonic flaw detection, vibration diagnostics, tensometry and others. The disadvantages of NDT-methods include their dependence on a large number of factors, the difficulty of excretion the main signal about the presence of a malfunction, and in some cases, low diagnostic accuracy.

The lifetime assessment results are gives out in statistical or deterministic form. The proposed work describes the procedure for assessing the lifetime in a deterministic form using the results of fatigue tests of laboratory samples and mathematical modeling of the investigated part. 


\section{PROBLEM ANALYSIS AND TASK STATEMENT}

The methods for calculating of machines lifetime based on the analysis of the service life of individual parts under non-stationary cyclic loading

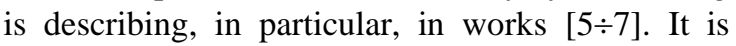
indicated that in conditions of a multiaxial stress state, the calculation should be performed using equivalent stresses, taking into account the gradient of their change.

At present, fracture mechanics approaches are also used to establish the lifetime, which belong to the group of extrapolative methods. For this purpose, use is made of the Paris equation $[8,9]$, or its improved model (Forman-Newman-de Koning), which is used in software systems NASGRO, AFGROW and others [10, 11]. These methods are based on fatigue testing of standard specimens made from the material of the investigated part, with sharp notches and of created fatigue cracks (or on tests of the part itself). The disadvantages of this group of methods:

- it is necessary to be possibility diagnose the presence of a crack;

- it is difficult to determine the geometric characteristics of the crack - the depth and shape of its front;

- the residual resource is installed only, i.e. the durability of object is determined.

The lifetime of a part, determined by the summary quantity of stress change cycles $N_{\Sigma}$ at the multilevel loading is most often assessed by a technique, which based on the linear damage summation hypothesis (LDSH): $\sum_{i=1}^{I} n_{i} / N_{i}=1$, where $n_{i}$ - the operating time at the $i$-th level; $N_{i}-$ durability to fracture along the fatigue curve at the same level; $I$ - the quantity of loading steps.

To implement of this technique must be given the equation of the fatigue curve and the stepwise cyclogram of unsteady loading, built in relative coordinates $\sigma_{1 i} / \sigma_{1 \max }-n_{i} / N_{\Sigma}$, where $\sigma_{1 i}-$ the first principal stress at the $i$-th level of the cyclogram; $\sigma_{1 \max }-$ its maximum value; $n_{i} / N_{\Sigma}-$ the relative duration of the stress $\sigma_{1 i}$. Such a cyclogram can be determined by one of the methods indicated in the introduction. Consequently, as the initial data, it is necessary to know the parameters of the fatigue curve and the sizes of a levels of the loading cyclogram - their height $\sigma_{1 i} / \sigma_{1 \max }$ and width $n_{i} / N_{\Sigma}$.

In the general case, the dependence of the cyclic durability $N$ on the value of the alternating first principal stress $\sigma_{1}$ is most often described by the power equation

$$
\sigma_{1}^{m} \cdot N=\sigma_{R}^{m} \cdot N_{G}=10^{C},
$$

where $m$ and $C$ are parameters; $\sigma_{R}$ - the endurance limit of the part at a given coefficient of asymmetry of the cycle $R ; N_{G}$ - the abscissa of the breaking point of the fatigue curve plotted in $\log \sigma-\log N$ coordinates.

The method for calculating $N_{\Sigma}$ is based on replacing the actual alternating loading mode, specified by a multilevel stress cyclogram, with an equivalent mode with constant stress $\sigma_{E}$ and the quantity of cycles before failure $N_{E}$. The maximum value of the first principal stress $\sigma_{E}=\sigma_{1 \max }$ is taken as the equivalent stress [12]. From the LDSH and equation (1), the condition for the equivalence of these modes follows

$$
\sum_{i=1}^{I} \sigma_{1 i}^{m} \cdot n_{i}=\sigma_{E}^{m} \cdot N_{E}
$$

The relationship between $N_{E}$ and $N_{\Sigma}$ is represented as

$$
N_{E}=\mu_{m} N_{\Sigma} .
$$

The equivalence coefficient by the cycles $\mu_{m}$ is determined taking into account equality (2)

$$
\mu_{m}=\sum_{i=1}^{I} \lambda_{i}^{m} \cdot\left(\frac{n_{i}}{N_{\Sigma}}\right),
$$

where $\lambda_{i}=\sigma_{1 i} / \sigma_{\max }=\sigma_{1 i} / \sigma_{E}-$ the relative first principal stress at the $i$-th level of the cyclogram.

The equivalent quantity of cycles is determined from equations (1) and (2) with $\sigma_{1}=\sigma_{E}$ by the formula

$$
N_{E}=10^{C} / \sigma_{E}^{m} .
$$

The lifetime of the part is determined from the formula (3)

$$
N_{\Sigma}=N_{E} / \mu_{m} \text {. }
$$

To calculate the lifetime (6) according to this method, the coefficient $\mu_{m}$ should be known. This coefficient is or assigned or calculated by the formula (4) in depending on the parameter $m$. In engineering calculations, this parameter usually lies in the range $m=3 \div 25$ [12]. For example, when cyclic bending of parts made of normalized and improved steels, $m=6$, for hardened steels, $m=9$, and for parts made of cast irons and non-ferrous metals, recommendations for the purpose of $m$ are not given. As a result of the conservatism of such recommendations, the resource value determined by formula (6) may differ for heavy mode by $1.3 \div 1.5$ times, for light - by $2 \div 2.5$ times.

A common disadvantage of all calculation methods for determining the resource of parts is their extrapolation orientation: the greater the calculated durability $N_{\Sigma}$, the greater the error of the result. The error in determining the calculated value of $N_{\Sigma}$ is considered acceptable if it does not exceed $50 \%$ at an accuracy of determining the endurance limit of up to $12 \%$. Therefore, the refinement of the lifetime calculation is an urgent task.

The aim of the work is to improve the computational methodology for assessing the lifetime of machine parts operating under uneven cyclic loading, according to the criterion of fatigue strength.

To achieve this goal, the following steps must be completed: 
a) determination of the endurance limit of the investigated part under a symmetric loading cycle according to the method of the statistical theory of similarity of fatigue failure (STSFF) using the results of fatigue tests of samples, as well as finding the parameters $m$ and $C$ for this part of its fatigue curve;

b) adjusting the endurance limit of the part for a given cycle of stress change, as well as setting new values for the parameters $m$ and $C$ for it;

c) determination of the maximum value of the first principal stress $\sigma_{1 \max }$ acting in the dangerous area of the part, which is taken as equivalent;

d) calculation of the equivalence factor by the cycles $\mu_{m}$ for a given loading cyclogram;

e) calculation of the lifetimes of the part.

As an example of using the proposed technique, the life of the cylinder block (CB) of an axial piston hydraulic machine (APHM) 210.25 is diagnosed under typical loading conditions. The possibility of a significant increase in the operating pressure in the hydraulic machine by the criterion of the $\mathrm{CB}$ fatigue strength is shown.

\section{METHOD FOR CALCULATING THE LIFETIME OF PARTS BASED ON FATIGUE TESTS OF SAMPLES}

The proposed improved method for calculating the lifetime is determined by the sequential execution of the steps $a-e$ listed in paragraph 2 and include the following procedures.

\subsection{Step $a$}

The endurance limit of the object under study (it denoted by the index $D$ ) with a symmetric loading cycle is calculated by the formula $[13,14]$

$$
\sigma_{-1 D}=\frac{\bar{\sigma}_{-1} K_{V} K_{A}}{2 K_{t} /\left(1+\theta^{-v_{\sigma}}\right)+1 / K_{F}-1} .
$$

The medium value of the endurance limit $\bar{\sigma}_{-1}$ is established from the results of testing smooth laboratory samples (usually with a diameter of $d_{0}=7.5 \mathrm{~mm}$ ). Coefficients of a effect of surface hardening $K_{V}$, anisotropy $K_{A}$, of a surface roughness $K_{F}$, stress concentration (theoretical) $K_{t}$ are assigned according to the recommendations of the standard [13]. For parts of complex shape, the coefficient $K_{t}$ is determined by methods of the theory of elasticity or finite elements. The coefficient of metal sensitivity to stress concentration and the influence of absolute dimensions $v_{\sigma}$ is calculated using the fatigue fracture similarity equation, which in logarithmic coordinates is determined by a linear relationship [12]

$$
\log (\xi-1)=-v_{\sigma} \log \theta+u_{P} \cdot S,
$$

where $S$ - the mean square deviation of the function $\log (\xi-1) ; u_{P}-$ the quantile of the normal distribution corresponding to the probability of destruction $P$.
In formula (8), the relative criterion for the similarity of fatigue fracture $[12,13]$

$$
\theta=\frac{L_{D} / \bar{G}_{D}}{L_{0} / \bar{G}_{0}},
$$

where the numerator $L / \bar{G}$ and denominator $L_{0} / \bar{G}_{0}-$ respectively the criteria for the similarity of fatigue failure of the object under consideration and a smooth laboratory sample with a diameter of $d_{0}=7.5 \mathrm{~mm} ; \bar{G}$ and $\bar{G}_{0}-$ the relative gradients of the first principal stress in the zone of its concentration for the part and the sample, respectively. The part parameter $L$ is understood as the length of the perimeter of the dangerous section or its part, within which the maximum stresses act, determined for the similarity equation (8) on the dependence

$$
\xi=\frac{\sigma_{\max }}{u}
$$

where $\sigma_{\max }=\sigma_{-1 D} \cdot K_{t}$ - the maximum value of stresses in the zone of their concentration; $u=0.5 \bar{\sigma}_{-1}-$ the minimum damaging stress, which is taken equal to half the endurance limit of a smooth laboratory sample [12]. At these values, formula (10) takes the form

$$
\xi=\frac{2 \sigma_{-1 D} \cdot K_{t}}{\bar{\sigma}_{-1}}
$$

The coefficient $v_{\sigma}$ is the tangent of the slope between the $\log \theta$ axis and the $\log (\xi-1)$ line. The sequence for calculating $v_{\sigma}$ is as follows. At first according to the results of tests or calculations, we find several pairs of values $\xi_{i}$ and $\theta_{i}$ for various standard sizes of samples or parts made from the material in question. Then is calculate the corresponding coordinates of the points $\log \theta_{i}$, $\log \left(\xi_{i}-1\right)$. Finally, according to the results of statistical processing by the least squares method, the regression line equation $\log (\xi-1)=v_{\sigma} \log \theta$ is determined.

Consequently, according to the results of fatigue tests of samples, the coefficient $v_{\sigma}$ is determined analytically and the endurance limit of the part is calculated using (7) at a symmetric stress cycle.

To find the parameters $m$ and $C$ of equation (1) of the fatigue curve of the part, the technique presented in [15] was used. Its essence lies in the fact that the results of fatigue tests of samples performed at a symmetric cycle of stress variation are subject to correction. The values stresses of test for each $j$-th sample $\sigma_{-1 N j}$ (at the obtained values for it $N_{j}$ ) are decrease on the difference

$$
\Delta \sigma=\bar{\sigma}_{-1}-\sigma_{-1 D} .
$$

The found stress values $\sigma_{-1 D N j}=\sigma_{-1 N j}-\Delta \sigma$ are subjected to linear regression analysis.

As a result, the parameters $m$ and $C$ of its fatigue curve are determined for the part under consideration at a symmetric stress cycle. At the obtained value of $m$ is used to calculate the 
coefficient $\mu_{m}$ (4), and at the value of $C$ - the equivalent number cycles $N_{E}(5)$.

\subsection{Step $b$}

If the stress cycle of a part has an arbitrary asymmetry coefficient $R$, then to calculate its endurance limit $\sigma_{R D}$ one can use any of the limiting amplitude diagrams (LAD) [16]. For example, one can apply Soderberg's LAD defined by the equation

$$
\frac{\sigma_{a}}{\sigma_{-1 D}}+\frac{\sigma_{m}}{\sigma_{0.2}}=1
$$

where $\sigma_{0.2}-$ conventional yield strength; $\sigma_{a}=0.5(1-R) \sigma_{R D} \quad$ and $\quad \sigma_{m}=0.5(1+R) \sigma_{R D} \quad-$ amplitude and average stresses of cycle.

At these values, from formula (13), we determine the endurance limit of the part, which is the maximum stress of cycle

$$
\sigma_{R D}=\frac{2 \sigma_{-1 D} \cdot \sigma_{0.2}}{(1-R) \sigma_{-1 D}+(1+R) \sigma_{0.2}} .
$$

To calculate the parameters of equation fatigue curve (1), it is necessary to set the limits of bounded endurance of the part $\sigma_{R D N j}$ at a given coefficient $R$. They are determined by formula (14), in which instead of the endurance limit $\sigma_{-1 D}$, the limits of bounded endurance $\sigma_{-1 D N j}$ should be taken

$$
\sigma_{R D N j}=\frac{2 \sigma_{-1 D N j} \cdot \sigma_{0.2}}{(1-R) \sigma_{-1 D N j}+(1+R) \sigma_{0.2}} .
$$

The values calculated according to (15) are subjected to linear regression analysis to determine the parameters $m$ and $C$ of the fatigue curve of the part with the asymmetry coefficient $R$. If the part undergoes a symmetrical loading cycle, then step 3.2 is not performed.

\subsection{Step $c$}

To assess the resource of the part, the equivalent stress is used, which is appoint equal to the maximum value of the first principal stress $\sigma_{E}=\sigma_{1 \max }$ $[12,17]$. For parts of simple forms (axles, shafts, etc.) $\sigma_{1 \max }$ is calculated quite simply. For parts of complex configuration, $\sigma_{1 \max }$ is determined by numerical methods, of which the most common is the finite element method (FEM). This method makes it easy enough to determine both $\sigma_{1 \max }$ and its gradient $[14,17,18]$.

\subsection{Step $d$}

Under the operating modes of the machine specified by the loading cyclogram and the calculated parameter $m$ of the fatigue curve of the part under consideration, the coefficient $\mu_{m}$ is calculated by the formula (4). However, for many classes of even the same type of equipment (agricultural machinery, transport, etc.) the operating modes are determined by the operating conditions and may differ significantly. With order to assess the lifetime, the real modes of loading such machines by methods of statistical processing are bring to five typical ones [19].
The integral distribution functions $\Phi k(\lambda)$ are used as a characteristic of loading under typical modes, where the index $k$ is the conditional number of the typical mode $(1-$ heavy, 2 - medium equiprobable, 3 - medium normal, 4 - light and 5 especially light).

The dimensionless argument of this function is the level of the relative variable load or the relative stresses proportional to it $\lambda=T / T_{\max }=\sigma_{1} / \sigma_{1 \max }$. Here $T$ and $T_{\max }$ - load values, for example, acting and maximum rotating or bending moments; $\lambda-$ the relative first principal stress arising in the dangerous area of the part under the action of these loads.

Integral representations of the functions $\Phi_{k}(\lambda)$ for typical loading modes and the calculation formulas obtained from them are given in Table 1.

In Table 1 denoted: $a, b, s-$ distribution parameters; $\lambda_{m}=1-\int_{0}^{1} \Phi_{k}(\lambda) d \lambda$ - medium value of the relative load (stress) for a typical modes; $\mathrm{B}(a, b)$ - Euler's beta-function; erf $(z)$ - the integral of probabilities.

The graphs obtained according to the calculation formulas of the Table 1 are shown in

\begin{tabular}{|c|c|c|}
\hline$k$ & $\begin{array}{l}\text { Statistical } \\
\text { characteristics } \\
\text { of the modes }\end{array}$ & $\begin{array}{l}\text { Function presentation } \\
\text { forms } \Phi_{k}(\lambda): \\
\text { integrals* and } \\
\text { calculation formulas }^{* *}\end{array}$ \\
\hline 1 & $\begin{array}{l}\beta \text {-distribution } \\
a=6.8 ; b=2 \\
\lambda_{m}=0.77\end{array}$ & $\begin{array}{l}\frac{1}{\mathrm{~B}(a, b)} \int_{0}^{\lambda} x^{a-1}(1-x)^{b-1} d x^{*}= \\
\quad=(7.8-6.8 \cdot \lambda) \cdot \lambda^{6.8 * *}\end{array}$ \\
\hline 2 & $\begin{array}{c}\text { Equiprobable } \\
\text { distribution } \\
\lambda_{m}=0.5 \\
\end{array}$ & $\int_{0}^{\lambda} d x^{*}=\lambda^{* *}$ \\
\hline 3 & $\begin{array}{c}\text { Normal } \\
\text { distribution } \\
s=0.19 ; \\
\lambda_{m}=0.5\end{array}$ & $\begin{array}{l}\frac{1}{s \sqrt{2 \pi}} \int_{-\infty}^{\lambda} \exp \left(-\frac{\left(x-\lambda_{3}\right)^{2}}{2 s^{2}}\right) d x^{*}= \\
=0.5[1+\operatorname{erf}(1.860807 \cdot(2 \lambda-1))]^{* *}\end{array}$ \\
\hline 4 & $\begin{array}{l}\beta \text {-distribution } \\
a=2.2 ; b=3 \\
\lambda_{m}=0.42\end{array}$ & $\begin{aligned} & \frac{1}{\mathrm{~B}(a, b)} \int_{0}^{\lambda} x^{a-1}(1-x)^{b-1} d x^{*}= \\
= & \left(6.72-9.24 \lambda+3.52 \lambda^{2}\right) \cdot \lambda^{2.2 * *}\end{aligned}$ \\
\hline 5 & $\begin{array}{l}\beta \text {-distribution } \\
a=1.8 ; b=4 \\
\lambda_{m}=0.31\end{array}$ & $\begin{array}{l}\frac{1}{\mathrm{~B}(a, b)} \int_{0}^{\lambda} x^{a-1}(1-x)^{b-1} d x^{*}= \\
=\left(8.512-16.42 \lambda+12.10 \lambda^{2}-\right. \\
\left.-3.19 \lambda^{3}\right) \lambda^{1.8 * *}\end{array}$ \\
\hline
\end{tabular}
figure 1 .

Table 1Typical loading modes

The traditional graphics representation of operating modes is obtained by changing the position of the coordinate axes: $\lambda$ - the ordinate, and $\Phi_{k}(\lambda)$ - the abscissa. This procedure is equivalent to rotating the graphs shown in figure 1 to the left by $90^{\circ}$ (fig. 2). In this case, the values of the abscissas $\Phi_{k}(\lambda)$ decrease from 1 to 0 , which is unconventional for graphs of functions. Therefore, 
a supplement axis of the relative quantity of cycles $n / N_{\Sigma}$ is introduced, having an increasing range from 0 to 1 . The argument $\Phi_{k}(\lambda)$ is related to the values of $n / N_{\Sigma}$ by the dependence

$$
n / N_{\Sigma}=1-\Phi_{k}(\lambda) \text {. }
$$

where $n$ is the quantity of cycles corresponding to the relative load.

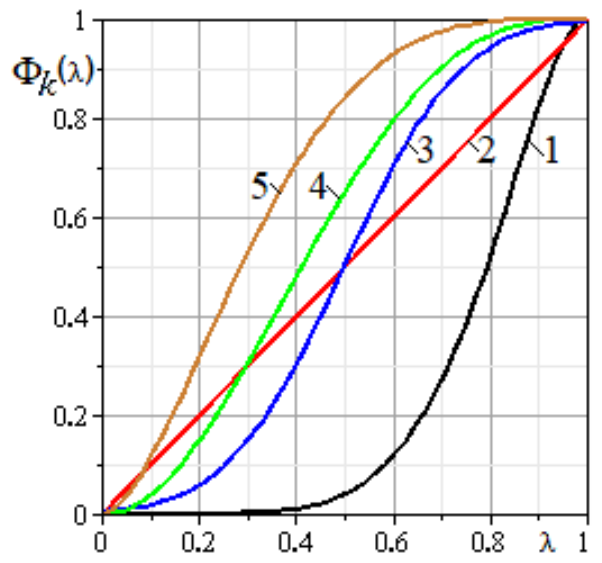

Fig. 1. Graphs of functions $\Phi_{k}(\lambda)$ for typical loading modes: 1 - heavy; 2 - medium equiprobable; 3 - medium normal; 4 - light; 5 - especially light

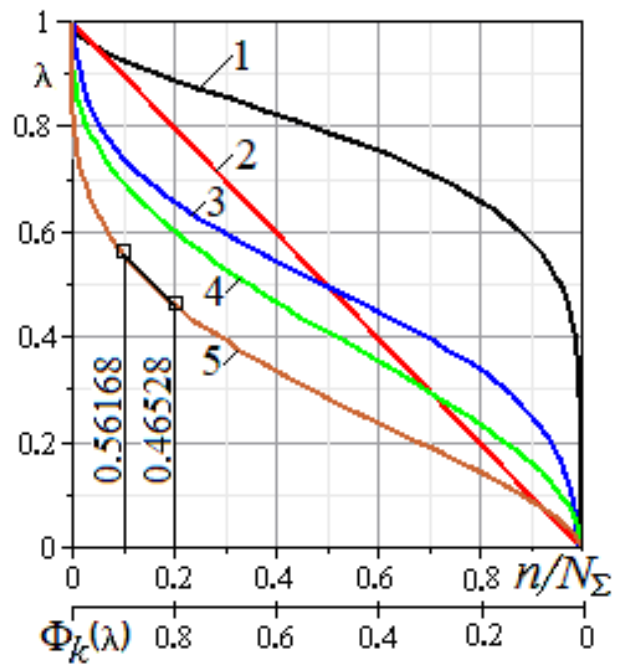

Fig. 2. Cyclograms of typical loading modes

It is not possible to obtain analytical expressions for describing the curves $\lambda\left(n / N_{\Sigma}\right)$ (except for line 2, for which $\Phi_{2}(\lambda)=\lambda$ and therefore $\left.\lambda=1-n / N_{\Sigma}\right)$. The ordinates of the remaining curves can be determined only discretely, numerically solving the equations $\Phi_{k}(\lambda)=n / N_{\Sigma}$. For example, for especially light mode 5 (Table 1) by solving the transcendental equation

$$
\left(8.512-16.42 \lambda+12.10 \lambda^{2}-3.19 \lambda^{3}\right) \lambda^{1.8}=n / N_{\Sigma} .
$$

The ordinates of the cyclogram are obtained, $\left.\lambda\right|_{n / N_{\Sigma}=0.1}=0.56168 ;\left.\lambda\right|_{n / N_{\Sigma}=0.2}=0.46528$.

To calculate the coefficient $\mu_{m}$ (4), it is necessary to construct a stepwise cyclogram loading equivalent to the accepted typical one (fig. 2). For this purpose, the argument $n_{i} / N_{\Sigma}$ is discretized with a step $\Delta_{n_{i} / N_{\Sigma}}$ and the values of $\lambda_{i}$ determined in the middle of each interval. The function $\lambda\left(n / N_{\Sigma}\right)$ has a significant gradient in some parts of the argument changes (fig. 2), therefore, to ensure the required accuracy, the specified step must be sufficiently small.

\subsection{Step $e$}

After execution of steps $3.1 \div 3.4$ of the described refined technique, the lifetime of the part is calculated by the formula (6).

\section{CALCULATIONS OF THE CB LIFETIME OF HYDRAULIC MACHINE}

As an example of using the proposed technique, the lifetime calculation of the CB APHM 210.25 (fig. 3) is performed.
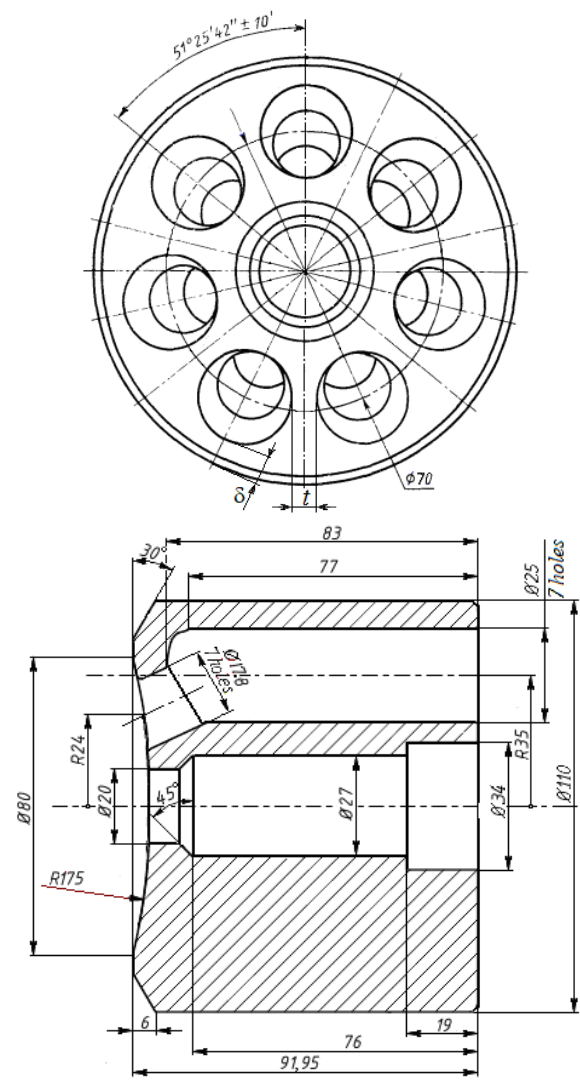

Fig. 3. Drawing CB of APHM 210.25

The increase in the energy efficiency of hydraulic machines is provided by an increase in the working pressure. Over the past $40 \div 50$ years, the working pressure has increased from $12 \div 16 \mathrm{MPa}$ to $25 \div 32 \mathrm{MPa}$, and this trend continues. An increase in pressure in the APHM leads to an increase in stresses in their parts. In result is increases the rate of accumulation of fatigue damage and reduces the life of those parts in which stresses change cyclically. As a result, the probability of failures increases, and about $30 \%$ of them fall on two main parts, the $\mathrm{CB}$ and the distributor $[17,20,21]$. Wear minimization is 
achieved by using antifriction materials for friction pairs and improving the quality of the working fluid (fine filtration, special additives, etc.), [22, 23]. Alloys of copper, bronzes and brasses, have high antifriction properties. At the same time, bronzes, especially tin ones, resist wear well. Therefore, the cylinder block was traditionally made of tin bronze CuSn12, in which the ultimate strength $\sigma_{u}=320 \mathrm{MPa}$, the conventional yield strength $\sigma_{0.2}=180 \mathrm{MPa}$ and the elongation at break $\delta=12 \%$ [14].

In APHM 210.25, the nominal discharge pressure is $p=16 \mathrm{MPa}$, and the drain pressure is $p_{0}=1 \mathrm{MPa}$ [19]. Due to the fact that $p_{0}<<p$ it is accepted $p_{0}=0$, then the asymmetry coefficient of the $\mathrm{CB}$ loading cycle will be $R=0$. Therefore, the calculation of the endurance limit $\mathrm{CB}$ is carried out in two steps 4.1 and 4.2.

\subsection{Calculation by step $a$}

To calculate the endurance limit CB using formula (7), the following values were obtained in [14]: $\quad \bar{\sigma}_{-1}=110 \mathrm{M \Pi а}, \quad K_{t}=1.28, \quad \theta=9.24, \quad K_{V}=1$, $K_{A}=0.9, K_{F}=0.99$.

The coefficient $v_{\sigma}$ characterizes the slope of line (8) to its abscissa axis. To calculate it, a linear regression analysis of pairs of numbers $\log \theta_{i}$ and $\log \left(\xi_{i}-1\right)$, obtained for objects made of $\mathrm{CB}$ material, bronze CuSn12, is performed, table 2 .

Table 2. Characteristics of the tested objects made of bronze CuSn12

\begin{tabular}{cccccc}
\hline $\begin{array}{c}\text { Object } \\
\text { types }\end{array}$ & $K_{t}$ & $\begin{array}{c}\sigma_{-1}, \\
\mathrm{MPa}\end{array}$ & $\begin{array}{c}\sigma_{\max } \text { by } \\
(10), \mathrm{MPa}\end{array}$ & $\theta$ & $\xi$ \\
\hline $\begin{array}{c}\text { Corset } \\
\text { sample [6] }\end{array}$ & 1.014 & 110 & 111.5 & 0.8785 & 2.028 \\
\hline $\begin{array}{c}\text { The } \\
\text { sample is } \\
\text { notched [6] }\end{array}$ & 1.737 & 70 & 121.6 & 0.1049 & 2.211 \\
\hline $\begin{array}{c}\text { CB APHM } \\
210.25\end{array}$ & 1.28 & 61.2 & 78.3 & 9.236 & 1.424 \\
\hline
\end{tabular}

If the probability $P=50 \%$, then the quantile $u_{P}=0$ and the regression line equation (8) has the form

$$
\log (\xi-1)=-0.2358 \cdot \log \theta \text {. }
$$

In this case, the value of the correlation coefficient $r=-0.912$ was obtained, which indicates a high connection between the function and its argument in equation (17). From this equation, the coefficient of metal sensitivity to stress concentration and to scale factor was found, $v_{\sigma}=0.2358$.

The established coefficient $v_{\sigma}$ made it possible, according to equation (7), to determine the endurance limit of the $\mathrm{CB}$ with a symmetric cycle of stress change, $\bar{\sigma}_{-1 D}=61.2 \mathrm{MPa}$.

\subsection{Calculation by step $b$}

In the process of operation, the stresses in the $\mathrm{CB}$ change almost under zero cycle, that is, $R=0$, therefore, the procedure of this stage must performed. According to the formula (14) calculated $\bar{\sigma}_{0 D}=91.3 \mathrm{MPa}$.

Let us determine for the $\mathrm{CB}$ at $R=0$ the values of the parameters $m$ and $C$ of equation (1). For this purpose, the above-described recalculation of the results of fatigue tests of samples corsetry type $\sigma_{-1 N j}$ according to formulas (12) and (15) is used. The difference according to the formula (12) is $\Delta \sigma=110-61.2=48.8 \mathrm{MPa}$ and the value $\sigma_{-1 D N j}=\sigma_{-1 N j}-48.8$. Formula (15) is reduced to the form $\sigma_{0 D N j}=\frac{2\left(\sigma_{-1 N j}-48.8\right) \cdot 180}{\sigma_{-1 N j}+131.2}$. The obtained pairs of values $\lg N_{j}, \lg \sigma_{0 D N j}$ were subjected to linear regression analysis and the parameters of equation (1) were determined, $m=8.384, C=24.04$ (correlation coefficient $r=-0.920$ ).

\subsection{Calculation by step $c$}

The design of the seven-piston CB APHM 210.25 is rather complicated (fig. 4), therefore, the calculation of its stress state was carried out by the finite element method (FEM) in the ANSYS Workbench software complex at a nominal pressure $p=16 \mathrm{MPa}$. It was found that the greatest stresses act in the inter-cylinder partition separating two axial holes loaded by the nominal pressure. The calculated maximum value of the first principal stress $\sigma_{1 \text { max }}=75.4 \mathrm{MPa}$ is taken as the equivalent stress $\sigma_{E}$ (fig. 4).

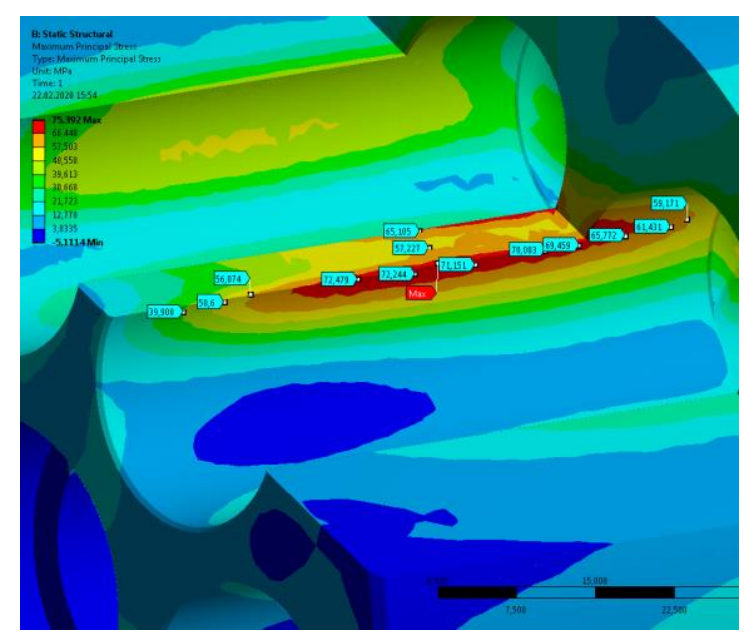

Fig. 4. Distribution of the first main stress in the longitudinal section of the inter-cylinder partition at a nominal pressure $p=16 \mathrm{MPa}$ in a seven-cylinder CB

\subsection{Calculation by step $d$}

APHM are used in many types of machine units and can work under various conditions loading. To determine the equivalence coefficient over the cycles $\mu_{m}$ (4) are must know: the parameter $m$ of the 
power-law equation (1) and the loading cyclogram presented in a stepwise form. The value $m=8.384$ was obtained in step 4.2. To calculate the coefficient $\mu_{m}$, the main typical modes of loading are considered (fig. 2). The calculations were performed for pressures $p=16 \mathrm{MPa}, p_{1}=20 \mathrm{MPa}$ and $p_{2}=25 \mathrm{MPa}$. The equivalent stress at $p=16 \mathrm{MPa}$ was obtained at step 4.3.

The maximum stresses $\sigma 1 \max$ arising in the $\mathrm{CB}$ at the indicated pressure levels do not exceed the elastic limit of bronze CuSn12, $\sigma_{e} \approx \sigma_{0.2}=180 \mathrm{MPa}$, therefore, the linear dependence for the function $\sigma_{E}(p)$ is valid. Therefore, it is possible to determine $\sigma_{E}$ at pressures $p_{1}$ and $p_{2}$ on the basis of a linear relationship between pressure and stress without using MKE:

$$
\begin{gathered}
\sigma_{E 1}=\sigma_{E} \cdot p_{1} / p=75.4 \cdot 20 / 16=94.2 \mathrm{MPa}, \\
\sigma_{E 2}=\sigma_{E} \cdot p_{2} / p=75.4 \cdot 25 / 16=117.8 \mathrm{MPa} .
\end{gathered}
$$

At these pressures, the obtained values of the coefficients $\mu_{m}$ are presented in Table 3 .

Table 3. Coefficients $\mu_{m}$ for typical loading modes CB made of bronze CuSn12

\begin{tabular}{lccc}
\hline \multirow{2}{*}{ Loading mode } & \multicolumn{3}{c}{ Discharge pressure } \\
\cline { 2 - 4 } & $16 \mathrm{MPa}$ & $20 \mathrm{MPa}$ & $25 \mathrm{MPa}$ \\
\hline Heavy & 0.2067 & 0.2153 & 0.2157 \\
\hline $\begin{array}{l}\text { Medium } \\
\text { equiprobable }\end{array}$ & 0.1010 & 0.1059 & 0.1064 \\
\hline Medium normal & 0.02800 & 0.03591 & 0.03687 \\
\hline Light & 0.01279 & 0.01778 & 0.01864 \\
\hline Especially light & 0.002375 & 0.003988 & 0.004566 \\
\hline
\end{tabular}

The relative first principal stresses $\lambda_{i} \leq 0.5$ are not damaging [12] and were not taken into account when calculating the coefficient $\mu_{m}$ (4), therefore the number of terms in formula (4) $I \neq$ const. Calculations have shown that the error in the value of $\mu_{m}$ does not exceed $1 \%$ at a sampling step of $\Delta_{n_{i} / N_{\Sigma}}=0.01$.

\subsection{Calculation by step $e$}

The equivalent quantity of cycles (5) is determined according to the parameters $m$ and $C$ calculated for the zero cycle of the $\mathrm{CB}$ operation at pressures of 16, 20 and $25 \mathrm{MPa}$.

$$
\begin{aligned}
& N_{E}=\frac{10^{C}}{\sigma_{E}^{m}}=\frac{10^{24.04}}{75.4^{8.384}}=1.996 \cdot 10^{8} \text { cycles, } \\
& N_{E 1}=\frac{10^{C}}{\sigma_{E 1}^{m}}=\frac{10^{24.04}}{94.2^{8.384}}=3.087 \cdot 10^{7} \text { cycles, } \\
& N_{E 2}=\frac{10^{C}}{\sigma_{E 2}^{m}}=\frac{10^{24.04}}{117.8^{8.384}}=4.737 \cdot 10^{6} \text { cycles. }
\end{aligned}
$$

For typical modes $(k=1 \div 5)$, the results of calculations of the lifetime $N_{\Sigma}$ according to the formula (6) are given in Table 4.

Increasing the working pressure from $16 \mathrm{MPa}$ to $25 \mathrm{MPa}$, i.e. approximately 1.5 times, leads to a sharp decrease in the CB lifetime $(50 \div 80$ times $)$.

In work [24], during the lifetime tests of the APHM 210.25 for CB at a pressure of $18 \mathrm{MPa}$ the value was set $N_{E}=6.3 \cdot 10^{7}$ cycles. This pressure determines the equivalent stress $\sigma_{E}=75.4 \cdot 18 / 16=84.8 \mathrm{MPa}$. Calculation according to the described method, formula (5), gives

$$
N_{E}^{(18)}=\frac{10^{C}}{\sigma_{E}^{m}}=\frac{10^{24.04}}{84.8^{8.384}}=7.453 \cdot 10^{7} \text { cycles } .
$$

This result differs from the experimental one [24] by $18 \%$, which is satisfactory for lifetime prediction [11].

Table 4. Lifetime CB (bronze CuSn12), $N_{\Sigma}$, million cycles

\begin{tabular}{lccc}
\hline \multirow{2}{*}{ Loading mode } & \multicolumn{3}{c}{ Lifetime at pressure $p$} \\
\cline { 2 - 4 } & $16 \mathrm{MPa}$ & $20 \mathrm{MPa}$ & $25 \mathrm{MPa}$ \\
\hline Heavy & 965.7 & 143.4 & 21.96 \\
\hline $\begin{array}{l}\text { Medium } \\
\text { equiprobable }\end{array}$ & 1976 & 291.5 & 44.52 \\
\hline Medium normal & 7129 & 859.6 & 128.5 \\
\hline Light & 15606 & 1736 & 254.1 \\
\hline Especially light & 84042 & 7741 & 1037 \\
\hline
\end{tabular}

At the nominal rotation frequency of the shaft of the APHM 210.25, equal to $n=3000 \mathrm{~min}^{-1}$, the CB lifetime in hours is calculate using the formula

$$
t_{\Sigma}=N_{\Sigma} /(60 \cdot n)
$$

For typical loading modes, the results of calculations according to (18) are given in Table 5.

Table 5. Lifetime CB (bronze CuSn12), $t \Sigma$, thousand hours

\begin{tabular}{lccc}
\hline \multirow{2}{*}{ Loading mode } & \multicolumn{3}{c}{ Lifetime at pressure $p$} \\
\cline { 2 - 4 } & $16 \mathrm{MPa}$ & $20 \mathrm{MPa}$ & $25 \mathrm{MPa}$ \\
\hline Heavy & 5.37 & 0.800 & 0.122 \\
\hline $\begin{array}{l}\text { Medium } \\
\text { equiprobable }\end{array}$ & 11.0 & 1.62 & 0.247 \\
\hline Medium normal & 39.6 & 4.78 & 0.714 \\
\hline Light & 86.7 & 9.65 & 1.41 \\
\hline Especially light & 467 & 43.0 & 5.76 \\
\hline
\end{tabular}

\section{SOME WAYS TO INCREASE THE CB LIFETIME ACCORDING BY FATIGUE STRENGTH CRITERION}

An increase in the resource can be achieved by improving the design of the $\mathrm{CB}$ and increasing the characteristics of the fatigue strength of the material. Here are indicated a number of possible ways.

\subsection{Constructive methods}

They are designed to reduce hazardous loads.

\subsubsection{Application of hydraulic unloading}

Flats are applied to the outer surface of the CB in areas where high stresses are applied. The flats are connected by radial channels with holes of cylinders. Outside onto the $\mathrm{CB}$ a steel pressure bandage is established. During the operation of the APHM, the working fluid enters the cavities formed between the flats and the bandage, providing a reduction in hazardous stresses [25]. 


\subsubsection{Changing the quantity of axial cylinders} (pistons)

The ICP size $t_{n}$ at an arbitrary number of axial cylinders $n_{c}$ and an outer wall thickness $\delta$ (fig. 3) is determined by the formula

$$
t_{n}=\left(D_{e}-d_{7} \cdot \sqrt{\frac{7}{n_{c}}}-2 \delta\right) \sin \frac{\pi}{n_{c}}-d_{7} \cdot \sqrt{\frac{7}{n_{c}}} .
$$

The CB of APHM 210.25 has the outer diameter $D_{e}=110 \mathrm{~mm}$, the diameter of the axial holes $d_{7}=25 \mathrm{~mm}, \delta=7.5 \mathrm{~mm}, t_{7}=5.37 \mathrm{~mm}$. Calculations using formula (19) for $n_{c}=5$ and $n_{c}=9$ give $t_{5}=8.87 \mathrm{~mm}$ and $t_{9}=2.97 \mathrm{~mm}$, respectively. Therefore, it is rational to reduce the number of axial cylinders to $n_{c}=5$, at which $t_{5}$ takes a maximum value. In the version of the $\mathrm{CB}$ design with $n_{c}=5$ a nominal pressure of $p=16 \mathrm{MPa}$, the maximum stresses are $46.7 \mathrm{MPa}$ in the ICP, which is $38.1 \%$ less than in the case of $n_{c}=7$. It should be noted that a decrease in the number of cylinders leads to an increase in pressure pulsation and may be undesirable for some hydraulic units.

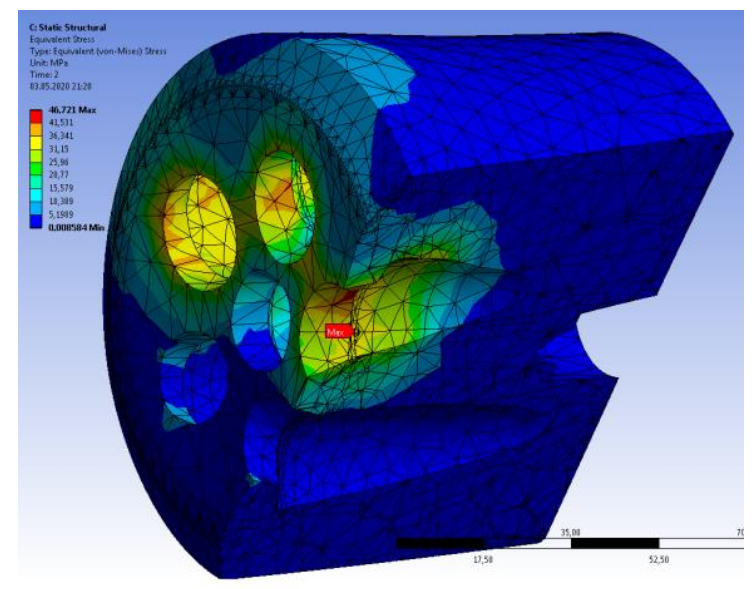

Fig. 5. Distribution of the first main stress in the longitudinal section of the inter-cylinder partition at a nominal pressure $p=16 \mathrm{MPa}$ in a five-cylinder CB

\subsubsection{Displacement of axes of axial cylinders bores in the radial direction}

The analysis of the performed calculation indicates that the stresses in the serial $\mathrm{CB}$ are distributed significantly unevenly: the stresses in the outer walls of the axial holes are insignificant, and their maximum occurs in the ICP, and similar situation is observed in the five-cylinder CB (fig. 5). To reduce the maximum stresses, it proposed to increase the thickness $t$ of the ICP (fig. 3) by increasing the radius $R_{c}$ of the location of the axial holes.

With an increase in $R_{c}$ to $37.5 \mathrm{~mm}$, the maximum stresses in the ICP decrease by $30.3 \%$ [18] and, accordingly, the CB lifetime increases.

\subsection{Technological methods}

Designed are to increase the endurance limit of the CB material.

\subsubsection{Hardening of CuSn12 bronze by hydroextrusion}

This hardening of cylindrical billets intended for the manufacture of CB was studied in [26]. Fatigue strength tests corseting samples at a extrusion ratio of $30 \%$ show an increase in the endurance limit from $\sigma_{-1}=110 \mathrm{MPa}$ to $\sigma_{-1}=200 \mathrm{MPa}$, i.e. by $81.8 \%$.

\subsubsection{Application of bimetal steel-bronze}

An increase in the $\mathrm{CB}$ resource is achieved by making it from steels that have higher fatigue resistance characteristics than copper alloys. To ensure wear resistance and reduce friction power losses, the contact surfaces CB from steel are clad with bronze or other antifriction materials [27, 28].

For example, it is proposed to use high-alloy high-speed steel $\mathrm{W} 18 \mathrm{Cr} 4 \mathrm{~V}$, with the endurance limit $\sigma_{-1}=270 \div 330 \mathrm{MPa}$. The given values of $\sigma_{-1}$ are the characteristics of smooth laboratory samples. It is recommended to recalculate on a steel CB and determine its resource according to the methodology proposed in this work. The effect on the lifetime of the facing antifriction material due to its small thickness can be disregarded. Currently, APHMs are manufactured with bimetallic CB by Sauer-Danfoss, Parker, Bosch Rexroth, Linde, etc. This allows you to increase the working pressure up to $48 \mathrm{MPa}$. It should be noted that the application of bimetallic $\mathrm{CB}$ is possible with the developed technology of their manufacture and operation, in case of violation of which the detachment of the antifriction coating is possible [29, 30].

\subsubsection{The use of high strength copper alloys}

For brass $\mathrm{Zn} 28 \mathrm{Mn} 3 \mathrm{Co} 2$ (ultimate strength $\sigma_{u}=595 \mathrm{MPa}$, conditional yield strength $\sigma_{0.2}=413 \mathrm{MPa}$ ), fatigue resistance tests of corset and notched samples were performed [31], the fatigue limits were established $\sigma_{-1 C}=230 \mathrm{MPa}$ and $\sigma_{-1 V}=140 \mathrm{MPa}$, respectively. The endurance limit $\sigma_{0 D}=226 \mathrm{MPa}$ was calculated for CB using formula (14), which is 2.2 times higher than the values for CuSn12. The calculation results for typical modes $(k=1 \div 5)$ are performed according to formulas (4), (6) and (18) and are presented in Tables $6 \div 8$.

Table 6. Coefficients $\mu_{m}$ for typical loading conditions $\mathrm{CB}$ made of brass $\mathrm{Zn} 28 \mathrm{Mn} 3 \mathrm{Co} 2$

\begin{tabular}{lccc} 
& \multicolumn{3}{c}{ CB made of brass Zn28Mn3Corge pressure } \\
\hline \multirow{2}{*}{ Loading mode } & $20 \mathrm{MPa}$ & $25 \mathrm{MPa}$ & $40 \mathrm{MPa}$ \\
\cline { 2 - 4 } & 0 & 0.0283 & 0.1347 \\
\hline Heavy & 0 & 0.0313 & 0.0737 \\
\hline Medium equiprobable & 0 & 0 & 0.0182 \\
\hline Medium normal & 0 & 0 & 0.00708 \\
\hline Light & 0 & 0 & 0.00114 \\
\hline Especially light & & & \\
\hline
\end{tabular}


Table 7. Lifetime CB (brass Zn28Mn3Co2), $N$, million cycles

\begin{tabular}{lccc}
\hline \multirow{2}{*}{ Loading mode } & \multicolumn{2}{c}{ Lifetime at pressure $p$} \\
\cline { 2 - 4 } & $20 \mathrm{MPa}$ & $25 \mathrm{MPa}$ & $40 \mathrm{MPa}$ \\
\hline Heavy & Not limited & $2.86 \cdot 10^{6}$ & $1.656 \cdot 10^{3}$ \\
\hline $\begin{array}{l}\text { Medium } \\
\text { equiprobable }\end{array}$ & Not limited & $2.58 \cdot 10^{6}$ & $3.045 \cdot 10^{3}$ \\
\hline Medium normal & Not limited & Not limited & $1.233 \cdot 10^{4}$ \\
\hline Light & Not limited & Not limited & $3.170 \cdot 10^{4}$ \\
\hline Especially light & Not limited & Not limited & $1.956 \cdot 10^{5}$ \\
\hline
\end{tabular}

Table 8. Lifetime CB (brass Zn28Mn3Co2), ts, thousand hours

\begin{tabular}{lccc}
\hline \multirow{2}{*}{ Loading mode } & \multicolumn{3}{c}{ Lifetime at pressure $p$} \\
\cline { 2 - 4 } & $20 \mathrm{MPa}$ & $25 \mathrm{MPa}$ & $40 \mathrm{MPa}$ \\
\hline Heavy & Not limited & 15883 & 9.3 \\
\hline $\begin{array}{l}\text { Medium } \\
\text { equiprobable }\end{array}$ & Not limited & 14360 & 16.9 \\
\hline Medium normal & Not limited & Not limited & 68.5 \\
\hline Light & Not limited & Not limited & 176.1 \\
\hline Especially light & Not limited & Not limited & 1094 \\
\hline
\end{tabular}

Also promising are high-strength copper alloys CuNi20Mn20, CuNi7SiCr with an endurance limit of $\sigma_{-1}=300 \mathrm{MPa} \quad[32], \quad \mathrm{Cu}-\mathrm{Ni}-\mathrm{Al}$ with $\sigma_{-1}=200 \div 290 \mathrm{MPa}$ [33] and $\mathrm{CuCrZr}$ with $\sigma_{-1}=170 \mathrm{MPa}[27]$.

\section{DISCUSSION OF RESEARCH RESULTS}

A method is proposed for assessing the lifetime of parts operating under unsteady cyclic loading, based on the provisions of the statistical theory of similarity of fatigue fracture. In the calculation, the transition from the fatigue curve of the specimens to the fatigue curve of the part is performed and the equivalent number of cycles $N_{E}(5)$ is determined, corresponding to the mode with constant stress $\sigma_{1 \max }$. Described is a calculation method for determining the equivalence coefficient by cycles $\mu_{m}$, using the parameter $m$ of the fatigue curve of the part without taking into account stresses at which damage does not accumulate. Based on the obtained values of $N_{E}$ and $\mu_{m}$, the calculated lifetime of the part $N_{\Sigma}$ are determined.

The developed method for assessing the resource of structural elements by the fatigue resistance criterion was used to analyze the operation the CB of APHM.

It was found that for a serial hydraulic machine 210.25 with a nominal pressure of $p=16 \mathrm{MPa}$, the lifetime under any loading conditions is more than 5000 hours. When pressure is forcing, the lifetime sharply decreases, in proportion to the value of $p^{9}$ (Table 5).Therefore, the use of APHM 210.25 at elevated $p$ values is not recommended.

With an increase in the working pressure, two directions of increasing the $\mathrm{CB}$ lifetime are proposed: constructive and technological. The purpose of the first direction is to reduce the level of dangerous stresses by improving the design. The proposed methods make it possible to reduce the maximum stresses in the $\mathrm{CB}$ by $30 \div 38 \%$. As a result, when forcing pressure $p$ to $25 \mathrm{MPa}$ under heavy loading, the lifetime increases from 122 hours to $7000 \div 22000$ hours. The purpose of the second direction is to increase the endurance limit of $\mathrm{CB}$ by strengthening traditional tin bronze, using high-strength copper alloys, or using a steel-bronze bimetal. CB made by such methods can operate at pressures up to $40 \mathrm{MPa}$ and even higher (Table 8).

In addition to the performed calculations, it will be expedient to investigate the possibility of increasing the operating pressure of the APHM by combining the two indicated directions of the $\mathrm{CB}$ modernization.

The lifetime calculations in this work are performed in a deterministic form. If is need a statistical analysis, can use the techniques from [14, 34].

\section{CONCLUSIONS}

The possibility of assessing the lifetime of a part by the criterion of fatigue strength at the design stage is shown. The calculation is based on the provisions of the fatigue fracture similarity theory and includes:

- a testing samples from the material of the part (at least two series with different stress concentrators for each) and determining their endurance limits;

- the calculation of the characteristics of the stress-strain state in the vicinity of the possible failure of the part at a given loading;

- a cyclogram of variable loading.

To verification of proposed method, a calculation of the resource of a part of a complex configuration (a block of cylinders of an axial piston hydraulic machine) was performed and its results were compared with the known field tests of this part [24].

Some technological and constructive methods of modernization of the $\mathrm{CB}$, which significantly increase its service life, have been investigated.

\section{REFERENCES}

1. Nikolenko I, Olejnichenko A. Substantiation of structure and parameters of hydraulic stands with recuperation of capacities for diagnostics of adjustable hydromachines. Diagnostyka. 2008; 3(47): 165-169.

2. Paulus A. Tribolayer formation on bronze $\mathrm{CuSn} 12 \mathrm{Ni} 2$ in the tribological contact between cylinder and control plate in an axial piston pump with swashplate design. 10th international Fluid Power Conference. 2016: 251-262.

https://core.ac.uk/download/pdf/236372989.pdf

3. Chmelko V, Kliman V, Garan M. In-time monitoring of fatigue damage. Procedia Engineering. 2015; 101: 93-100.

https://core.ac.uk/download/pdf/82276386.pdf 
4. Hu Changhua, Zhou Zhijie, Zhang Jianxun, Si Xiaosheng. A survey on life prediction of equipment. Chinese Journal of Aeronautics. 2015; 28(1): 25-33.

5. https://doi.org/10.1016/j.cja.2014.12.020.

6. Będkowski W. Assessment of the fatigue life of machine components under service loading - a review of selected problems. Journal of Theoretical and Applied Mechanics. 2014. 52(2): 443-458.

7. Kurek A, Koziarska J, Łagoda T. The influence of the strain and stress gradient in determining strain fatigue characteristics for oscillatory bending. Materials. 2020;13(1):15. https://doi.org/10.3390/ma13010173.

8. Saintier N, Palin-Luc T, Benabes J, Cocheteux F. Non-local energy based fatigue life calculation method under multiaxial variable amplitude loadings. International Journal of Fatigue. 2013; 54: 68-83. https://doi.org/10.1016/j.ijfatigue.2012.12.013.

9. Tomaszek H, Jasztal M, Zieja M. Application of the Paris formula with $m=2$ and the variable load spectrum to a simplified method for evaluation of reliability and fatigue life demonstrated by aircraft components. Eksploatacja i Niezawodnosc Maintenance and Reliability. 2013; 15(4): 297-304.

10. Weizhen Song, Zhansi Jiang, Hui Jiang. Predict the fatigue life of crack based on extended finite element method and SVR. AIP Conference Proceedings 1967. 2018; 8. https://doi.org/10.1063/1.5039052

11. Maierhofer J, Gänser H-P, Simunek D, Leitner M, Pippan R, Luke M. Fatigue crack growth model including load sequence effects - Model development and calibration for railway axle steels. International Journal of Fatigue. 2020;132. https://doi.org/10.1016/j.ijfatigue.2019.105377

12. Wei Zhang, Qiang Wang, Xiaoyang Li, Jingjing He. A simple fatigue life prediction algorithm using the modified NASGRO Equation. Mathematical Problems in Engineering. 2016;8. https://doi.org/10.1155/2016/4298507.

13. Kogaev VP, Makhutov NA, Gusenkov AP. Calculations of machine elements and structures for strength and durability: Handbook (in Russian). Moscow, Mashinostroyeniye Publ. 1985; 224.

14. GOST 25.504-82 Calculations and strength tests. Methods for calculating fatigue resistance characteristics (in Russian).

15. Khomyak Yu, Kibakov O, Medvedev S, Nikolenko I, Zheglova V. Endurance limit of the axial-piston hydraulic machine cylinder block. Diagnostyka. 2020;21(1):71-79. https://doi.org/10.29354/diag/116691

16. Konoplev AV, Kononova OM, Kibakov OG. Refinement of the coefficient of relative durability for objects with low endurance limits (in Russian). Vesnik ONMU. 2018; 3(56): 197-205.

17. Gutyrya SS, Medvedev SA, Khomiak YuM, Chanchin AV. Probabilistic analysis of fatigue durability of an epicycle of a wheel gearbox of the trolleybus. Bulletin of NTU "KhPI". Series: Problem of mechanical drive. -Kharkiv : NTU "KhPI". 2017; 25(1247): 37-43.

18. Zheglova V, Khomiak Yu, Medvedev S, Nikolenko I. Numerical and analytical evaluation of service life of the details axial piston hydraulic machines with complicated configuration under cyclic loading.
Procedia Engineering. 2017; 176: 557-566. https://doi.org/10.1016/j.proeng.2017.02.298

19. Nikolenko IV, Khomyak YM, Zheglova VM, Kibakov OG, Medvedev SO. The design of responsible details of an axial piston hydraulic machine improving. IOP Conf. Series: Earth and Environmental Science. 2020; 408 012006: 12.

20. Reshetov DN, Ivanov AS, Fadeev VZ. Machine reliability (in Russian). Moscow, Graduate School Publ. 1988; 238.

21. Karpenko M, Bogdenvičius M. Reviev of energysaving technologies in modern hydraulic drives. Civil and transport engineering, aviation technologies. 2017;9(5):553-558. https://doi.org/10.3846/mla.2017.1074

22. Larchikov I, Yurov A, Stazhkov S, Grigorieva A, Protsuk A. Power analysis of an axial piston hydraulic machine of power-intensive hydraulic drive system. Procedia Engineering. 2014; 69: 512-517. https://doi.org/10.1016/j.proeng.2014.03.020

23. Rydberg K-E. Energy efficient hydraulics - system solutions for minimizing losses. National Conference on Fluid Power, Linköping, Sweden. 2015: 10.

24. Schuhler G, Jourani A, Bouvier S, Perrochat J-M. Multi technical analysis of wear mechanisms in axial piston pumps. Journal of Physics: Conference Series, 2017; 843: 012077. https://doi.org/10.1088/17426596/843/1/012077.

25. Dodin LG. Methods of testing axial-piston hydraulic machines (in Russian). Proceedings of VNIIStroydormash. 1981; 92: 23-29.

26. Dashchenko AF, Nikolenko VI. Calculation of the nominal pressure of axial piston hydraulic machines according to the geometric parameters of the pumping units with hydraulic unloading (in Russian). Proceedings of the Odessa Polytechnic University. 2005; 2 (24): 46-52.

27. Biryukov BN, Medvedev SA, Stanislavsky VG, Kibakov OG, Dobrinsky GK, Shemper LI, Vysotsky EN, Saakyants VP. Strengthening of bronze blocks of cylinders of axial piston hydraulic machines by the method of hydroextrusion. Construction and road building machinery. 1990; 4 (412): 13-14. (in Russian).

28. Mishnev R, Shakhova I, Belyakov A, Kaibyshev R. Deformation microstructures, strengthening mechanisms, and electrical conductivity in a $\mathrm{Cu}-\mathrm{Cr}-$ $\mathrm{Zr}$ alloy. Materials Science and Engineering: A. 2015;629(1):29-40. https://core.ac.uk/download/pdf/323218042.pdf

29. Shengrong Guo, Jinhua Chen, Yueliang Lu, Yan Wang, Hongkang Dong Hydraulic piston pump in civil aircraft: Current status, future directions and critical technologies. Chinese Journal of Aeronautics. 2020;33(1):16-30. https://doi.org/10.1016/j.cja.2019.01.013

30. Quan Ling-xiao, Cao Yuan, Luo Hong-liang, Guo Rui, Guo Haixin. Fatigue analysis of the cylinder in the axial piston pump. Conference: Fluid Power and Mechatronics (FPM). International Conference at Harbin. 2015; Conference paper: 8.

31. D'Andrea D, Epasto G, Bonanno A, Guglielmino E, Benazzi G. Failure analysis of anti-friction coating for cylinder blocks in axial piston pumps. Engineering Failure Analysis. 2019; 104: 126-138. https://doi.org/10.1016/j.engfailanal.2019.05.041 
32. Nikolenko IV, Khomyak YM, Kibakov AG. Calculation of the durability the cylinder block of hydraulic machines (in Russian). Bulletin of Machine Building. 1988; 2: 26-29.

33. Altenberger I, Kuhn H-A, Müller HR, Mhaede M, Gholami-Kermanshahi M, Wagner L. Material properties of high-strength beryllium-free copper alloys. International Journal of Materials and Product Technology. 2015; 50(2): 124-146. https://doi.org/10.1504/IJMPT.2015.067820

34. Chakrabarti A, Sarkar A, Saravanan T, Atikukke N, Sandhya R, Jayakumar T. Influence of mean stress and defect distribution on the high hycle fatigue behaviour of cast Ni-Al bronze. Procedia Engineering. $\quad 2014 ; \quad 86$ : 103-110. https://doi.org/10.1016/j.proeng.2014.11.017

35. Nieslony A, Böhm M. Mean stress effect correction in frequency-domain methods for fatigue life assessment. Procedia Engineering. 2015; 101: 347 354. https://doi.org/10.1016/j.proeng.2015.02.042

Received 2021-08-26

Accepted 2021-11-14

Available online 2021-11-20

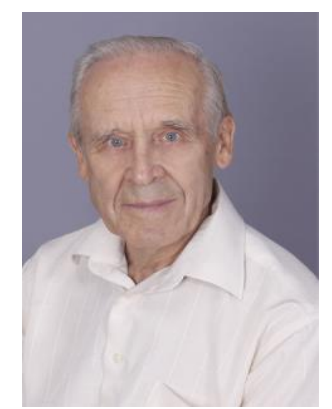

Yuriy KHOMIAK has received his $\mathrm{PhD}$ in Technical Sciences in 1980. Currently he is assistant professor of Department of metalcutting machine tools, metrology and certification in Odessa Polytechnic State University

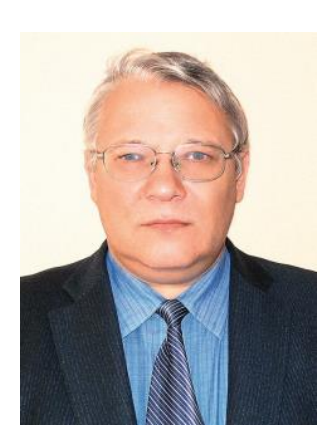

Oleksandr KIBAKOV has received his $\mathrm{PhD}$ in Technical Sciences in 1994. Professor, currently he is Dean by Faculty of Port Engineering of Odessa National Maritime University.

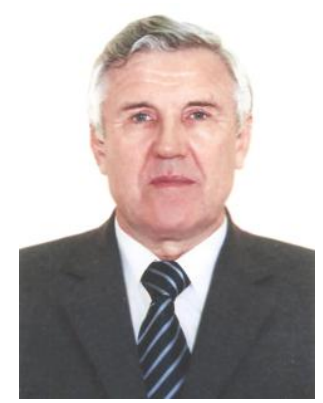

Stanislav MEDVEDEV has received his $\mathrm{PhD}$ in Technical Sciences in 1981. Currently he is assistant professor of "Machine Science" Department in Odessa National Maritime University.

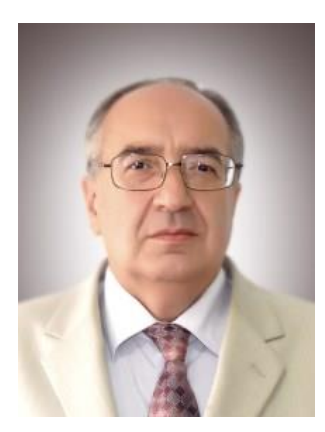

Ilya NIKOLENKO has received his Dr.Sn in Technical Sciences in 2006. Professor, currently he is Head of Department "Water supply, sanitation and sanitary equipment" in Academy of Construction and Architecture "V.I. Vernadsky Crimean Federal University".

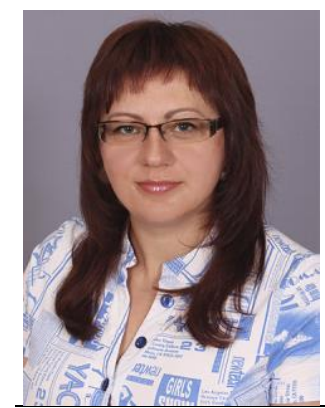

Victoria ZHEGLOVA has received his $\mathrm{PhD}$ in Technical Sciences in 2015. Currently he is assistant professor of Department of metalcutting machine tools, metrology and certification in Odessa Polytechnic State University. 